\title{
Cryo-TEM simulations of amorphous radiation-sensitive samples using multislice wave propagation.
}

\author{
Benjamin A. Himes and Nikolaus Grigorieff \\ Howard Hughes Medical Institute, RNA Therapeutics Institute, The University of \\ Massachusetts Medical School, Worcester, MA
}

\section{Abstract:}

Image simulation plays a central role in the development and practice of highresolution electron microscopy, including transmission electron microscopy of frozenhydrated specimens (cryo-EM). Simulating images with contrast that matches the contrast observed in experimental images remains challenging, especially for amorphous samples. Current state-of-the-art simulators apply post hoc scaling to approximate empirical solvent contrast, attenuated image intensity due to specimen thickness, and amplitude contrast. This practice fails for images that require spatially variable scaling, e.g., simulations of a crowded or cellular environment. Modeling both the signal and the noise accurately is necessary to simulate images of biological specimens with contrast that is correct on an absolute scale. The "Frozen-Plasmon" method is introduced which explicitly models spatially variable inelastic scattering processes in cryo-EM specimens. This approach produces amplitude contrast that depends on the atomic composition of the specimen, reproduces the total inelastic mean free path as observed experimentally, and allows for the incorporation of radiation damage in the simulation. These improvements are quantified using the matched-filter concept to compare simulation and experiment. The Frozen-Plasmon method, in combination with a new mathematical formulation for accurately sampling the tabulated atomic scattering potentials onto a Cartesian grid, is implemented in the open-source software package cisTEM.

\section{Introduction:}

The power (variance) of the noise in cryo-EM images outweighs the power of the signal, often by a factor of 20 or more. The dominant source of noise in cryo-EM is "shot" noise, arising from the stochastic nature of detecting an electron at a given location and time due to low-dose imaging conditions. A detailed analysis by Baxter et al [1] demonstrated the need to also consider structural noise, defined as any contrast arising from sources other than the final object of interest: carbon film, crystalline ice, radiation damaged particles, unwanted macromolecular conformers, the supporting amorphous ice etc. Unlike shot noise, structural noise is affected by objective lens aberrations, which give rise to the contrast transfer function (CTF). Baxter et al. 
modelled both the structural noise and the shot noise as additive white Gaussian noise, which fails to capture the artifacts and challenges that are commonly encountered during image processing, as previously demonstrated by Scheres et al [2].

An improvement in how the structural noise is simulated, particularly that arising from the supporting amorphous ice, can be found in TEM simulator [3] and inSilicoTEM [4]. They implement multislice wave propagation as described originally by Cowley and Moodie [5], resulting in noise that is affected by the CTF. The result of a multislice simulation is a probability distribution defined by the squared complex modulus of the electron wave function at the detector $\psi_{d}(x, y)$. The simulated image is then formed by drawing from a Poisson distribution unique to every pixel while incorporating the influence of the detector quantum efficiency (DQE).

Most of the information transferred from the specimen to the image in high-resolution cryo-EM is captured in phase contrast arising from interference between elastically scattered and non-scattered electrons. Amplitude contrast is also present due to electrons scattered outside the objective lens aperture, and loss of electrons from the elastic image due to inelastic scattering. The latter source of amplitude contrast is enhanced using an energy filter [6]. Amplitude contrast cannot be explained by linear image formation theory [7] and is accounted for post hoc via a phase shift term added to the CTF applied to the simulated image [8]. This treatment is also common practice in solving the inverse problem of image reconstruction, which seeks to answer the question "what is the probability of the model given the observed data." However, in forward modeling, which asks "what is the probability of observing some data given a particular model," it would be desirable to account for the fact that amplitude losses depend both on atom type, and on local mass thickness. For example, heavy atoms like gold scatter more electrons outside the objective lens aperture than light atoms. On the other hand, light atoms produce more amplitude losses in energy filtered images than heavy atoms due to their higher ratio of inelastic to elastic scattering [9][10].

The multislice formalism is essential for thick specimens where the projection approximation fails, as it incorporates important effects like multiple scattering of electrons and the curvature of the Ewald Sphere. Increasingly thick samples are also less transparent to electrons, and all simulators we are aware of apply an implicit "energy filter" to remove inelastically scattered electrons from the final image. To account for inelastic losses, a single thickness parameter is used to attenuate the image intensity according to 
where $I_{0}$ is the unattenuated image intensity, and $\lambda$ is the inelastic mean free path for single scattering - the average distance an electron passes through the specimen before scattered inelastically at least once. It is clear that these single filters cannot work for specimens with variable mass thickness, e.g., at the edge of a cell, for variable atomic composition, e.g., the increased phosphorus concentration in the nucleus, and even for many single particle samples [11].

Even with a limited subset of atomic species, to which we will constrain the following discussion, there are two very different environments that need to be simulated - the molecule and the solvent. We will refer to how well the molecule stands out from the solvent as "solvent signal-to-noise-ratio or $S N R_{\text {solvent }}$ as quantified by Yonekura et al [6] where $\bar{I}$ is the mean image intensity and $\sigma_{\text {solvent }}$ is the standard deviation in the solvent region.

$$
S N R_{\text {solvent }} \equiv \frac{\left|\bar{I}_{\text {protein }}-\bar{I}_{\text {solvent }}\right|}{\sigma_{\text {solvent }}}
$$

\section{0}

Typically, the solvent is added on top of the simulated molecules in projection, with a single value given by the mean inner potential for aqueous water. This approach, which we will refer to as "the continuum model", is equivalent to using an infinite time average of a collection of moving water atoms. One shortcoming of the continuum model is the failure to account for the hydration radius of a molecule, which should be zero inside a particle, higher than the bulk solvent immediately outside the particle envelope and gradually falling off with distance [12]. Ignoring the fact that molecules displace the solvent has been shown to produce $S N R_{\text {solvent }}$ that fails even visual inspection at exposures of $100 \mathrm{e}^{-} / \AA^{2}[4]$.

We now know that the infinite time average used in the continuum model does not adequately describe reality; even though the solvent is frozen low-density amorphous ice (LDA), it is not static during the imaging process. McMullan and Henderson quantified the motion of water molecules in LDA during imaging, estimating a RMSD of $\sim \frac{1 \AA}{\mathrm{e}^{-} / \AA^{2}}$ [13]. Importantly, this motion results in a blurring of the solvent over time, which can be thought of as low pass filtering, and so $\sigma_{\text {solvent }}$ decreases with increasing exposure. The net result is that $S N R_{\text {solvent }}$ is a function of the total exposure in an image, gradually increasing as the solvent becomes more blurred. Of note, the increase of $S N R_{\text {solvent }}$ with exposure is further amplified in experimental images by mass loss, which also decreases $\sigma_{\text {solvent }}$ and increases the numerator in Equation 2 by reducing $\bar{I}_{\text {solvent }}$. A more sophisticated version of our solvent model may implement this mass loss in future work. 
111 While $S N R_{\text {solvent }}$ is useful for its simplicity, a more detailed analysis requires another

112

113

114

115

116

117

118

119

120

121

122

123

124

125

126

127

128

129

130

131

132

133

134

135

136

137

138

139

140

141

142

143

144

145

146

metric to quantify how well the macromolecules we simulate match experimental images. For this, we propose using the matched filter, which is the statistically optimal realization of a cross-correlation detector. With image statistics characteristic of cryoEM data, the output of the matched filter can be simply defined as the ratio of the crosscorrelation coefficient (CCC) to the standard deviation of the CCC when only noise is present $\left(\sigma_{n}\right)$ [14] including any sources of structural noise as defined above.

$$
S N R_{m f}=\frac{C C C}{\sigma_{n}}
$$

The upper bound on the $S N R_{m f}$ is given by the ratio of the power of the input signal to the power of the noise in the image [15]. This means, for example, that a larger molecule will generally have a higher $S N R_{m f}$, while any disagreement between the signal in the image and the simulated template reduces the $S N R_{m f}$ from this maximal value. As such, the relative accuracy to which the simulated molecular density matches experimental data can be determined by searching images using a matched filter. To evaluate Equation 3, we use the cross-correlation tools and relevant preprocessing as available in cisTEM [16][17].

To understand why the continuum model fails to produce accurate $S N R_{\text {solvent }}$ it is instructive to consider the source of the motion of the solvent upon exposure to the imaging electrons. Along with radiation damage to the molecule of interest, sample motion is the result of energy being transferred to the specimen via inelastic scattering. For the samples in which we are interested, inelastic scattering is generally attributed to plasmons, i.e., collective excitation of valence electrons by the electric field of the imaging electrons. However, the extent to which these are bulk plasmons, which are strongly delocalized, or more localized single-electron excitations remains unclear [18]. Independent of the exact form of the plasmons, their net effect is an alteration of the system's Hamiltonian during imaging, such that product of a traditional multislice simulation, $\psi_{d}(x, y)$, is no longer valid. Just as the original multislice method introduced a division of the specimen potential into thin spatial slices to ensure the small angle approximation is valid, we suggest dividing the simulated exposure into small temporal slices, where the specimen does not change too much. While we refer to time here, what is most practical from the microscopist's point of view is exposure measured in $\mathrm{e}^{-} / \AA^{2}$. Therefore, the time step in our simulator is specified as the desired exposure per movie frame. Exposure rate dependent phenomena like detector DQE and beam coherence are parameterized by an exposure rate with the exposure time implicitly set by the software according to the user supplied exposure-per-frame divided by the 
exposure rate. The DQE parameterization is based on the results of Ruskin et al [19] while the coherence is parameterized based on the beam brightness of an Xfeg as given in the Titan Condenser Manual [20].

\section{Theory:}

There are three main components in modeling the image formation process in highresolution transmission electron microscopy (HRTEM) of which cryo-EM is a subset:

1. The relativistic electron wave function and its modulation by the sample.

2. The exposure-dependent Coulomb potential of the specimen.

3. The microscope, including apertures, detector, lens optics and aberrations.

In this work, we are concerned primarily with how the Coulomb potential changes due to energy being deposited in the specimen during imaging and will provide only a brief summary of the other two components. The interested reader is referred to, in increasing order of completeness, the treatments by Vulović et al [21], Kirkland [22], Reimer and Kohl [23] Hawkes and Kasper [24].

Unlike photons, electrons have a spin quantum number and so their interaction with matter is governed by solutions to the Dirac equation. Given reasonable approximations [24], a relativistically corrected version of the Schrödinger wave equation, called the Klein-Gordon equation is used in practice.

$$
\left[-\frac{\hbar^{2}}{2 m} \nabla^{2}+\mathcal{V}(\boldsymbol{r})\right] \psi(\boldsymbol{r})=E\left(\frac{m_{0}}{m}\right)\left[1+\frac{E}{2 m_{0} c^{2}}\right] \psi(\boldsymbol{r})
$$

Analytical solutions to this equation are intractable for all but the simplest systems, so we turn to multislice wave propagation [25], which produces an approximate numerical solution to this equation. The first step in a multislice simulation is the calculation of the specimen's Coulomb potential $\mathcal{V}(\boldsymbol{r} ; t)$. The time dependence will be omitted assuming a quasi-stationary solution for an exposure to a single electron. The potential is divided into thin slices along the imaging axis, which can be approximated by two-dimensional scattering potentials through which the electron wave function is sequentially propagated. This subdivision ensures the potential varies slowly in the direction of the electron wave propagation, such that the small angle approximation remains valid and scattered spherical wave fronts may be approximated locally by a parabola (Fresnel diffraction.) In the limit of infinitely thin slices, this results in an exact numerical solution to the Klein-Gordon equation [26]. 
Multislice simulations can model both elastic and inelastic scattering processes, provided that the respective Coulomb potentials can be calculated. In analogy to the optical potential in light microscopy, inelastic scattering is incorporated into the wave theory via a complex term in the specimen potential as introduced by J.C. Slater in 1937 [27].

$$
\mathcal{V}(\boldsymbol{r})=\mathcal{V}(\boldsymbol{r})_{\text {elastic }}+\mathrm{i} \mathcal{V}(\boldsymbol{r})_{\text {inelastic }}
$$

The isolated atom superposition approximation states that the specimen potential $\mathcal{V}(\boldsymbol{r})$ may be represented as the sum of the individual atomic potentials $\varphi(r)_{i}$. We introduce a scaling factor $\beta$ to compensate for the contribution of bonds among those atoms to maintain the correct total scattering cross section:

$$
\mathcal{V}(\boldsymbol{r}) \cong \beta \sum \varphi(\boldsymbol{r})_{\mathrm{i}}
$$

The elastic atomic potential can be calculated using relativistic Hartree-Fock wave functions [28]. The solutions for isolated atoms, having isotropic distributions, are commonly parameterized by a sum of four or five Gaussian functions [29]. Typically, the potential is recorded indirectly as these fits are tabulated as elastic electron scattering factors, defined as the Fourier transform of the elastic potential [30].

$$
f^{(e)}(\theta)=\frac{2 \pi m e}{h^{2}} \mathcal{F}\{\varphi(\boldsymbol{r})\}
$$

The numerator describes the product of the electron charge and relativistic mass, $h$ is the Planck constant, and $\mathcal{F}$ denotes the Fourier transform operator. An important relation we will return to later equates the spectral distribution of the scattering factor to the differential scattering cross section - the probability of an electron being scattered through a solid angle $\Omega$ :

$$
\frac{\mathrm{d} \sigma}{\mathrm{d} \Omega}=\left|f^{(e)}(\theta)\right|^{2}
$$

$$
\text { While } \mathcal{V}(\boldsymbol{r})_{\text {elastic }} \text { is straightforward to calculate from first principles, } \mathcal{V}(\boldsymbol{r})_{\text {inelastic }} \text { is more }
$$
problematic given the varied mechanisms with which an incident electron may transfer energy to the specimen: ionization, excitation, dissociative attachment, vibrational and rotational excitations, bremsstrahlung, etc. [31]. One example where $\mathcal{V}(\boldsymbol{r})_{\text {inelastic }}$ is well defined is for radiation-insensitive crystalline specimens, where thermal diffuse scattering (TDS) caused by phonon excitation is the primary contributor to the complex potential [29]. One model to calculate the TDS potential treats the time average atomic displacement through Debye-Waller factors and improves the accuracy of dynamic RHEED calculations [32]. This time average approach is analogous to how the solvent 
211 calculation, specimen motion, radiation damage and alignment errors are accounted for

212 in HRTEM simulations of biological specimens by B-factors, which are related to Debye-

213 Waller factors by a factor of 4.

214 While this time-averaged approach preserves the total intensity of the projected

215 interaction potential [33], it is well known that the image contrast produced in this way is

216 systematically wrong, often by a factor of three or more. The error, known as the Stobbs

217 factor [34], becomes worse with an increasing strength of the electron specimen

218 interaction, which in turn, depends on the average mass thickness in the specimen.

219

220 Stobbs et al. proposed two likely causes for the observed contrast mismatch between simulation and observation: a) existing simulators do not account properly for radiation damage to the specimen, and/or b) they fail to model the inelastic scattering with sufficient accuracy. As recently shown empirically, these are related phenomena [35].

Van Dyck et al. demonstrated that the Stobbs factor could be largely corrected for by using the "frozen phonon" method [36][37]. The approach is conceptually simple: A series of simulations are carried out where each atom is displaced randomly based on empirical TDS values. The intensities in the image plane as calculated from these individual simulations are then averaged together. Here we propose a similar idea, applied to radiation-sensitive frozen-hydrated specimens, where plasmons are the primary form of inelastic scattering. The "frozen plasmon method" presents several computational and theoretical challenges:

1. The number of solvent atoms $\left(\mathrm{O}\left(10^{9}\right)\right)$ greatly outweighs those of the macromolecules we wish to simulate $\left(\mathrm{O}\left(10^{5}\right)\right)$, requiring careful algorithmic design to make the computations tractable.

2. The solvent and macromolecules have very different elastic and inelastic total scattering cross-sections, as well as different average mass densities $(\sim 0.94$ $\mathrm{g} / \mathrm{cm}^{3}$ for low-density amorphous ice and $\sim 1.38 \mathrm{~g} / \mathrm{cm}^{3}$ on average for proteins) [38]. This means that the amplitude contrast and inelastic losses cannot be applied ad hoc to the final simulated image and must be considered on a peratom basis.

3. The preceding points also place a requirement on the accuracy of the calculation of each atomic scattering potential, which can no longer simply be rescaled and so must be correct from the start.

4. The scattering factor for plasmons in low-density amorphous ice is needed to achieve the appropriate contrast, which depends on the appropriate spectral distribution. To obtain an expression $\phi^{\text {(inelastic) }}(\boldsymbol{r})_{i}$, we start from the double 
differential scattering cross-section for plasmons [39]. The essential form is Lorentzian

$$
\frac{\mathrm{d}^{2} \sigma}{\mathrm{d} \Omega \mathrm{dE}} \propto \frac{1}{\theta^{2}+\theta_{E}^{2}}
$$

with the angular dependence $\theta$ and the energy dependence captured in the characteristic angle

$$
\theta_{\mathrm{E}}=\frac{m \omega_{\text {plasmon }}}{\hbar \boldsymbol{k}_{\mathbf{0}}^{2}}
$$

$\hbar \omega_{\text {plasmon }}$ is the energy loss of the plasmon, and $\boldsymbol{k}_{\mathbf{0}}$ the incident electron's wave vector. To calculate a scattering factor for plasmons, we first form an empirical probability distribution for plasmons arising from singly scattered electrons in amorphous ice, derived from EELS published by Du and Jacobsen [40]. We then numerically integrate Equation 8 over energies in the low-loss spectrum (7.5 $100 \mathrm{eV}$ ) for each angle.

We then combine this spectrum with empirical measurements of the ratio of inelastic to elastic total scattering cross sections, which are inversely proportional to atomic molecular weight [10]. As we calculate the elastic potential during simulation, we separately accumulate an inelastic potential scaled per atom by these total probabilities. During wave function propagation, this inelastic potential is given the correct Lorentzian form, taken to be the square root of the values above.

Plasmons scatter strongly at low angles and are generally referred to as being delocalized. This is reflected in Figure 1 where the inelastic scattering factor we derived for plasmons is compared to the elastic scattering factor for a glutamine molecule. While plasmon scattering dominates at low resolution compared to elastic scattering, it still contributes significantly at high angles as can be seen by the red hash marks in Figure 1 that demarcate bins of $20 \%$ total inelastic scattering probability. The precise nature of inelastic scattering in amorphous materials is not well understood, such that the relationship between this high-resolution information and the underlying specimen structure is not defined. 


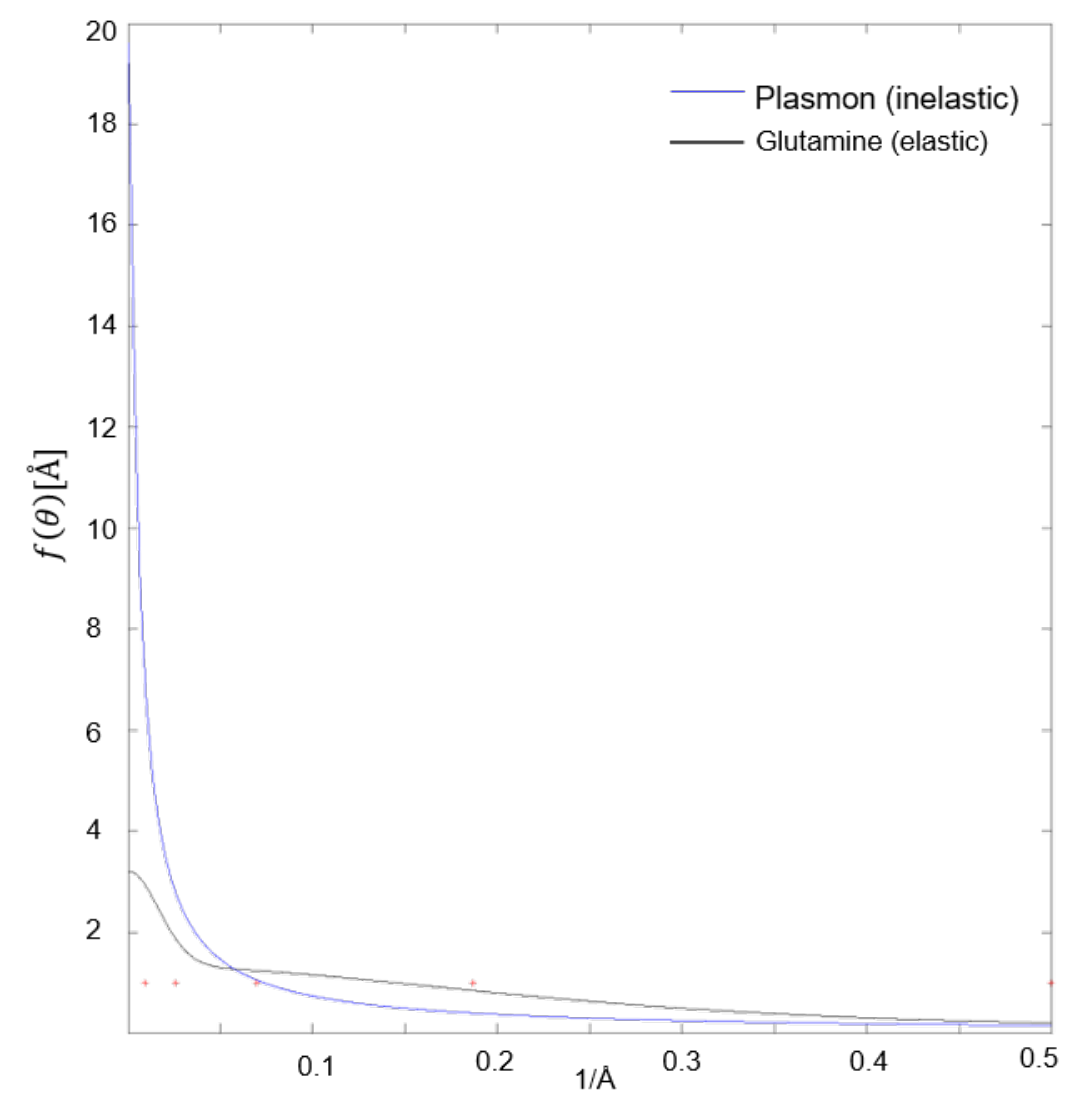

Figure 1 - Comparison of elastic scattering factor for a glutamine molecule (black line) vs. the inelastic scattering factor for a plasmon pseudo-particle (blue line.) Red markers indicate the right edge of bins comprising $20 \%$ total scattering probability for the inelastic Plasmon scattering factor as calculated from the squared norm of the scattering factor.

Results:

Accurate representation of molecular density:

282 For isolated neutral atoms, the scattering potential, defined as the Fourier transform of the parameterized scattering factors, can be written as

$$
\phi(\mathbf{r})=\frac{\hbar^{2}}{2 \pi \mathrm{m}_{0} \mathrm{e}} \sum_{i} \mathrm{a}_{i}\left(\frac{4 \pi}{\mathrm{b}_{i}+\mathrm{B}}\right)^{\frac{3}{2}} \exp \left[\frac{-4 \pi^{2}\left(\left(\mathrm{x}-\mathrm{x}_{0}\right)^{2}+\left(\mathrm{y}-\mathrm{y}_{0}\right)^{2}+\left(\mathrm{z}-\mathrm{z}_{0}\right)^{2}\right)}{\mathrm{b}_{i}+\mathrm{B}}\right]
$$

285 This atomic potential is sharply peaked in real space, requiring a high sampling rate 286 when discretizing in order to maintain the total projected potential. This high sampling 
rate effectively produces a numerical integration of Equation 10. To allow for coarser sampling, and hence improve the efficiency of our simulator, we analytically integrate the expression from Equation 10.

resulting in

$$
\phi(\mathbf{r})=\frac{\hbar^{2}}{2 \pi m_{0} e} \sum_{i} a_{i}\left(\frac{4 \pi}{b_{i}+B}\right)^{\frac{3}{2}} \prod_{j} \int_{0}^{\infty} d x_{j} \exp \left[\frac{-4 \pi^{2}\left(x_{j}-x_{j 0}\right)^{2}}{b_{i}+B}\right]
$$

While the potential in each voxel is marginally more complex to calculate (to evaluate the limits of integration, the error function must be evaluated six times per voxel, compared to a single exponential) this is more than compensated by the reduced number of voxels needed. For example, simulating at $0.5 \AA$ voxel pitch is $125 x$ less computationally expensive than simulating at $0.1 \AA$ voxel pitch. While the voxel pitch is the same in the $z$-dimension, the slab thickness is a free parameter which also affects computational efficiency. A simple test to determine the maximum allowable thickness, as suggested by Kirkland [22], is to search for the point where the results of the simulation become dependent on slab thickness. Our simulations begin to show a dependence on slab thickness around $7 \AA$ (data not shown) and, therefore, we typically use $5 \AA$. Even more important than computational speed, using Equation 12 in our simulations also means the sampled potential still has the correct magnitude and is not simply proportional to the continuous potential, as discussed in the following section.

\section{Compensating for the isolated atom superposition approximation:}

While the integral formulation of the scattering potential preserves the calculated potential of all the individual atomic contributions, there is still a systematic

underestimation of the scattering potential due to bonding interactions. This is generally estimated to be between $5-10 \%$ of the total potential [41], and ignoring this difference is referred to as the isolated atom superposition approximation. Given that we want to obtain images that are quantitative on an absolute scale, we sought to measure and calibrate this error. To approximate the redistribution of the scattering potential due to bonding in a biological specimen, we use the available data for amorphous carbon 
comparing to results from electron holography as follows. The average phase shift in a material depends on the mean inner potential of the material $\left(V_{0}\right)$, the thickness $(t)$, and an interaction constant $C_{E}$ [23].

$$
C_{E}=\frac{2 \pi}{\lambda} \frac{E_{0}+E}{2 E E_{0}+E^{2}}
$$

Additionally, surface boundary effects are also known to be important in cryo-EM imaging, so we compared our calculated phase shift $(\delta \varphi)$ to empirical results obtained using electron holography, which measures both the mean inner potential of carbon $\left(V_{0}=9.04 \mathrm{eV}\right.$ for $1.75 \frac{\mathrm{g}}{\mathrm{cm}^{3}}$ density $)$ and an additional thickness-independent surfaceinduced phase shift $\varphi_{\text {add }}$ (0.497 radians)[42].

$$
\delta \varphi=C_{E} V_{0} t+\varphi_{a d d}
$$

Considering the principle of a Zernike phase plate, we simulated an amorphous carbon sheet that should produce a phase shift of $\pi / 2$ radians (Figure 2A) with a density of $1.75 \frac{\mathrm{g}}{\mathrm{cm}^{3}}$ and $348.6 \AA$ thickness per Equation 14. Our simulation suggested that the average phase shift is $\sim 3.8 \%$ too small. To correct for this error, we introduce a constant scaling factor $(\beta)$ of 1.038 to the isolated atomic potentials. The simulated phase plate also serves as a sanity check that the calculation of the elastic scattering potential is consistent across different pixel sizes (Figure 2B).
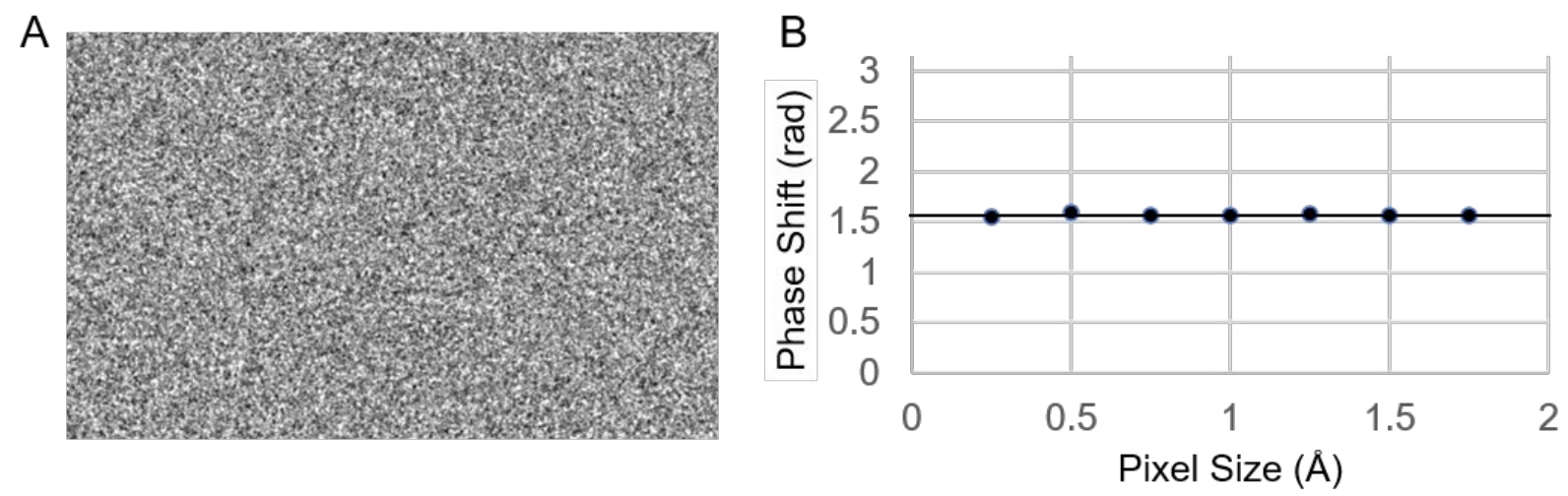

Figure 2 -A) "phase plate" simulated from amorphous layer of carbon atoms $348.6 \AA$ thick with a density of $1.75 \mathrm{~g} / \mathrm{cm}^{3}$ B) Mean phase shift for the simulated phase plate as a function of pixel sampling rate during simulation. Black line plotted at $\pi / 2$ radians for visual reference. 


\section{Modeling the solvent envelope}

340

341

342

343

344

345

346

347

348

349

350

351

352

353

354

355

356

357

358

359

360

361

362

363

364

365

366

367

368

369

370

371

372

373

374
In the previous section, we demonstrated that we could accurately calculate the contrast for a collection of randomly distributed atoms of a given density. Next, we show that in order to accurately compare a simulated protein density to experimental data, we must also consider the solvent displaced by the protein. This creates a low-resolution "hole" that impacts subsequent analysis as discussed in detail by Zhang and Sigworth [12]. We incorporate their hydration radius model into the simulator by tracking the smallest distance to any non-solvent molecule and weighting any nearby solvent with a probability distribution defined by normalizing Equation 1 from their paper. (We note that the parameter "r3" in table 1 of Zhang and Sigworth should be $\sim 3.0$, not 1.7, personal communication.)

When simulating isolated molecules to use for comparison to experimental images, we weight the average water potential by this probability distribution, with an exponential decay beyond $4 \AA$. This exponential decay is added because our knowledge of the sample rapidly decays to zero beyond the particle of interest. This produces an effect similar to the ad hoc model suggested previously by Henderson and McMullan [43]. When simulating images, the probability distribution is applied to individual pseudowater molecules as described next.

\section{Modeling radiation damage}

As soon as the electron beam is "switched on" the sample begins to accumulate radiation damage. This has long been known to be the limiting factor in cryo-EM [44] and an analytical function describing the effects of radiation damage as a Fourier space filter - $\xi(\boldsymbol{q})$ - was described by Grant and Grigorieff [45]. Since radiation damage is specimen dependent, the analytical model of Grant and Grigorieff will only strictly apply to rotavirus VP6 capsid protein, and not to nucleic acids, for example. Alternatively, radiation damage combined with other errors, for example uncorrected motion blur, may be fit using exposure-dependent B-factors [46][47]. The latter approach has the advantage that it does not try to separate the blurring due to radiation damage from other sources of blurring, which can be difficult in practice.

To quantify the accuracy in modeling radiation damage using the analytical model, $\xi(\boldsymbol{q})$, we employ the matched filter concept which is sensitive to the spectral distribution of the template when the noise does not have a flat power spectrum. Using the rotavirus DLP images originally used by Grant \& Grigorieff to derive their radiation damage model, we investigated several sources of such noise including the water shell, as described in the 
next section, the detector modulation transfer function (MTF), CTF coherence envelope, residual intra-frame motion, atomic modeling uncertainty and defocus uncertainty.

By looking at a single early frame from each of 18 movies, each with $\sim 1.5 e^{-} / \AA^{2}$ total exposure ( $0.77 e^{-} / \AA^{2}$ intra frame exposure), we could quantify how well we modelled these sources of noise, while assuming no significant radiation damage. In Figure 3A we show the projected scattering potential of our simulated DLP (PDB $3 \mathrm{gzu}$ ) along with the average $S N R_{m f}$ from 180 DLPs overlaid. Each source of noise is included cumulatively, showing the relative improvement in detection as the model becomes increasingly more detailed. Applying additional positive or negative B-factors only made the score worse. To illustrate the effect of each of these modifications on the template's spectral distribution, we also plot the ratio of the rotationally averaged power spectra of each simulation to the previous one. A representative image for frame 2 is shown in Figure $3 B$, while an average of frames $2-91$ is shown in Figure $3 \mathrm{C}$.

To assess the radiation damage model, we averaged movie frames $2-\mathrm{N}$ such that the accumulated exposure ranged from $10-100 e^{-} / \AA^{2}$ either with, or without, exposure filtering applied to the images. We then measured the $S N R_{m f}$ of the DLPs in these two sets of images using two sets of references calculated with, and without, exposure filtering applied during simulation, and plotted the results in Figure 3D. We found the largest increase in $S N R_{m f}$ using a total exposure of $50 e^{-} / \AA^{2}$ when applying the exposure filter to both the image and during simulation of the reference. To account for blurring due to residual intra-frame specimen motion in this experiment, the exposure filter was additionally modified to include a damping envelop which, for uniform motion, is trivial to show is a sinc function. Less trivial is determining the intra-frame specimen motion $\left(\boldsymbol{d}_{\boldsymbol{i}}\right)$ for which we only know a lower bound, estimated as the average of the displacements between the two neighboring frames, and applied as a sinc modulation along with the exposure filter (if included) for that frame.

$$
\frac{1}{N-1} \sum_{i=2}^{N} \operatorname{sinc}\left(\boldsymbol{q} \cdot \boldsymbol{d}_{\boldsymbol{i}}\right) \xi(q)_{i}
$$

Taken together, these results suggest that other modifications to the template that result in a better match to the experimental data can further improve the $S N R_{m f}$; for example, some amino acid side chains are affected more strongly by radiation damage than others, e.g., aspartate and the disulfide bond of cystine. These details could be incorporated into a new atom specific damage model in future work. 
A
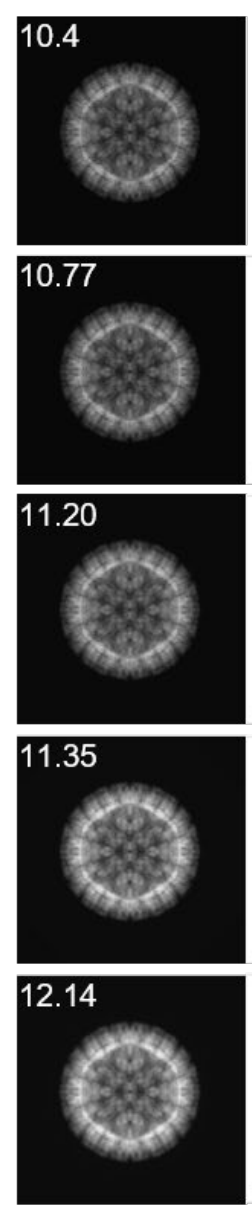

B

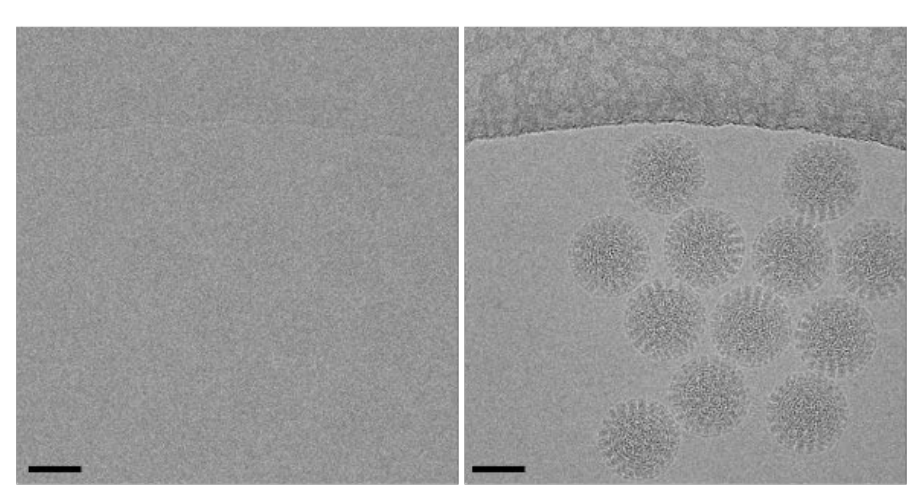

D

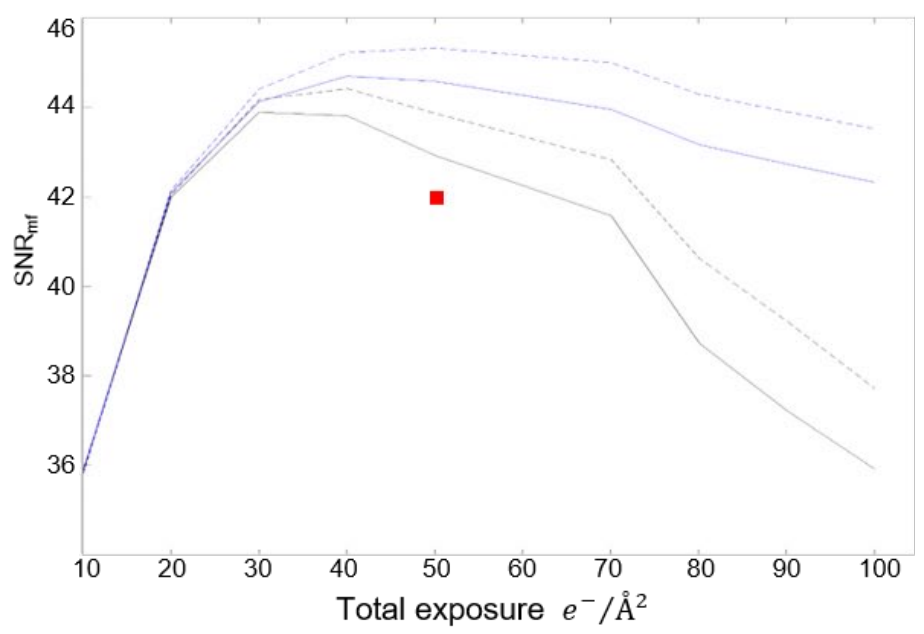

C
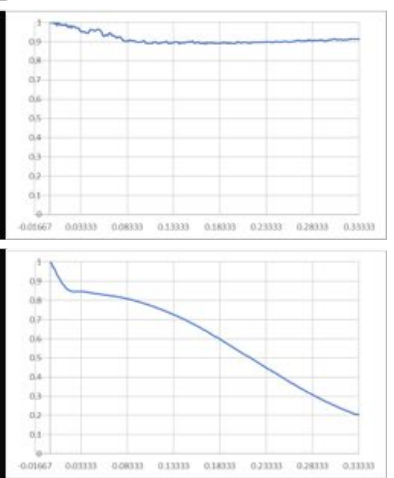

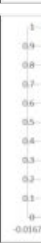

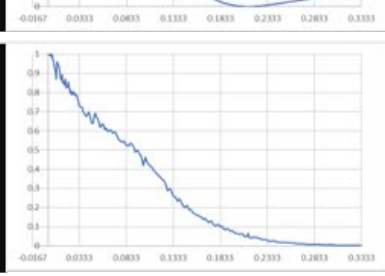

Figure 3 - A) Left column, projections of simulated rotavirus DLPs from PDB-3gzu with each of the blurring process applied cumulatively. Average peak $S N R_{m f}$ from 180 DLPs over 18 images overlaid in white. Right column, the shape of the individual envelopes produced by the respective blurring as calculated by dividing the rotationally averaged power spectrum by the preceding image. B) Example image from frame 2 and C) frames 2-91 of DLPs from the data set used in this analysis, kindly shared by Dr. Tim Grant. D) Average SNR values as a function of total exposure, frames averaged from 2-N, where $\mathrm{N}$ is on the total exposure on the horizontal axis. Solid black line, no filtering. Dashed black line, image summed from exposure weighted movie frames. Solid blue line, reference simulated with cumulative exposure filter as would normally be applied to a movie. Dashed blue line, both image and reference exposure filtered. Red square is the maximum SNR obtained by using a single B-factor to represent all the envelopes in (A). Scale bars $500 \AA$. 
412 Because the number of water molecules is very large, we elected to calculate a coarse-

413 grained model for water, where each water molecule is represented as a single,

414 isotropic scattering center. We based the scattering factor for our pseudo-waters on the

415 elastic scattering factor tabulated for oxygen, but scaled by the ratio of the total elastic

416 scattering cross-section of oxygen:water, which we know from experiment [31]. These

417 pseudo-molecules are seeded randomly at the proper density for low density

418 amorphous ice $\left(\sim 0.94 \mathrm{~g} / \mathrm{cm}^{3}\right)$. A movie is then simulated, where each time step (movie

419 frame) is defined by a user-specified exposure and the specimen is held constant within

420 that time. The simulated probability density for the constant potential is shown in Figure

421 4A while our coarse-grained all-atom model is shown in Figure 4B. The average

422 intensity in the solvent region is the same in both images.

423 In Figure 4D we show selected time points from a movie simulated using the continuous

424 solvent potential model (top row), the coarse-grained all-atom model (middle row), and

425 experimental data in the bottom row (EMPIAR-10061 [48]). As can be seen visually, the

$426 S N R_{\text {solvent }}$ is stronger for the continuum model, because the potential only has a DC

427 component. To quantify this effect, we calculated $S N R_{\text {solvent }}$ as in Equation 2, defining

428 the solvent region by the white portion of the mask in Figure 4C and the protein as the

429 central black region. The results are plotted in Figure 4F where the final $S N R_{\text {solvent }}$ is

430 about a factor of two too strong using the continuum model, while our model closely

431 matches that of experimental data. 


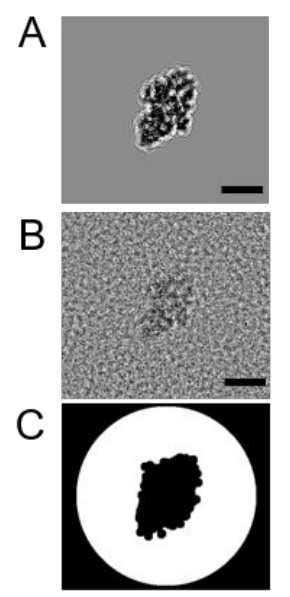

$\mathrm{F}$

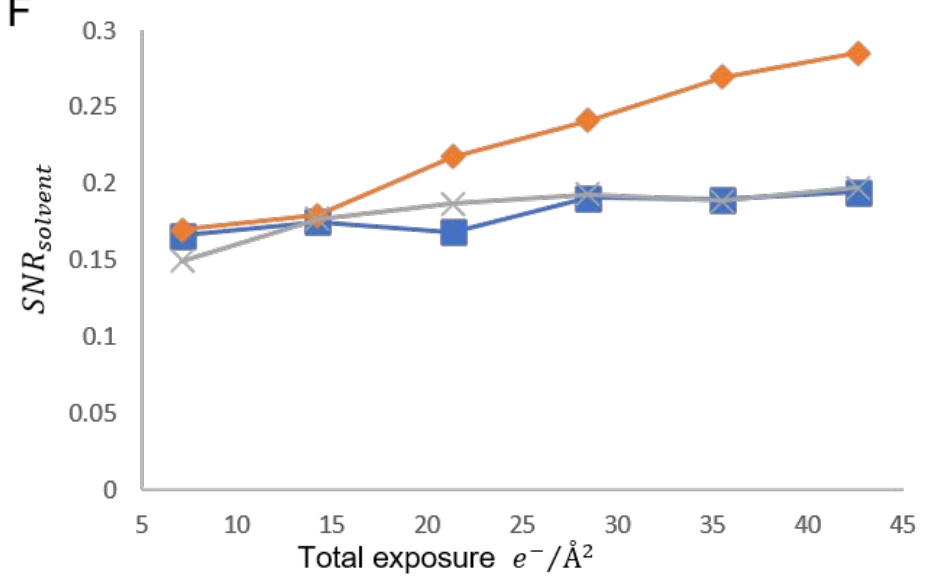

D
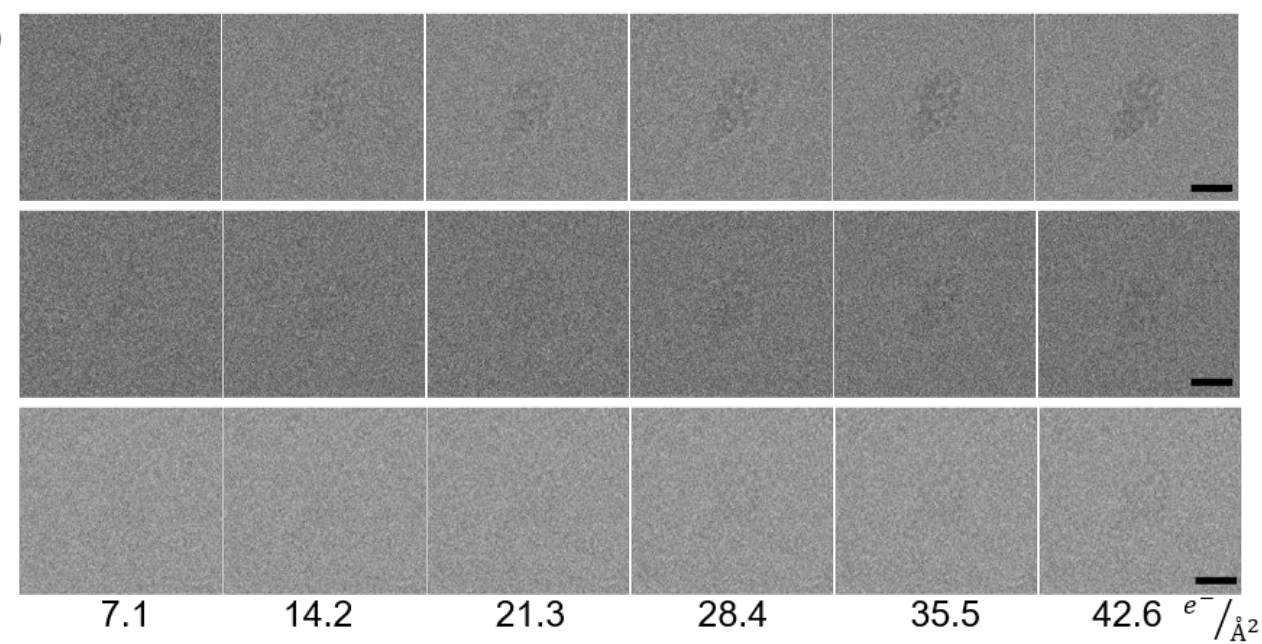

Figure 4 - Comparing the continuum and coarse-grained all-atom solvent model to experimental data. A) $\left|\Psi_{\text {detector }}\right|^{2}$ with a constant potential added for the solvent. B) $\left|\Psi_{\text {detector }}\right|^{2}$ with coarse-grained all-atom water model. The average intensity in A and B in the solvent region is identical within numerical precision. C) Mask used in calculating $S N R_{\text {solvent }}$ (equation 2 main text) where the white region was used for the solvent, and the central black region for the protein. D) Plot of $S N R_{\text {solvent }}$ as a function of accumulated exposure for experimental data (Blue line, square marker) coarse-grained all-atom solvent model (grey, $x$ marker) and constant solvent potential (orange, diamond marker). E) Images used in calculating (D) with the same color/marker scheme, and total exposure indicated along the bottom. Experimental data taken from EMPIAR-10061, beta-galactosidase. Scale bars $100 \AA$

\section{Amplitude contrast}

Amplitude contrast can arise from electrons being scattered outside the objective lens aperture, or by removing inelastically scattered electrons using an energy filter. The former is incorporated by applying an aperture function directly to the complex wave function prior to image formation, which results in an attenuation of the expected number of electrons at the detector. This is demonstrated in Figure 5 for a series of 
aperture diameters and a simulated amorphous specimen with density and thickness as used previously for the "phase plate" with atomic potentials of either carbon (orange circles), phosphorous (grey x's) or gold (blue squares). The smallest aperture used $(0.01 \mu)$ excludes all but the unscattered beam and so is a measure of total

444 transmittance of the simulated layer.

Amplitude losses due to inelastic scattering are incorporated into the multislice formalism via a complex scattering potential, commonly defined as linearly proportional to the real (elastic) potential, for example as in inSilicoTEM. A detailed analysis of why

448 this proportional model is inadequate is found in Dudarev et al. [29]. Our atomically specific inelastic scattering potential is described in the theory section. To demonstrate that it produces the correct amplitude losses, we rearrange Equation 1 to plot the

451 negative-natural logarithm of the expected electron count vs solvent thickness. Fitting 452 this with linear regression gives a readout of the simulated inelastic mean-free path in 453 Figure 6 which closely matches experimental numbers [49].

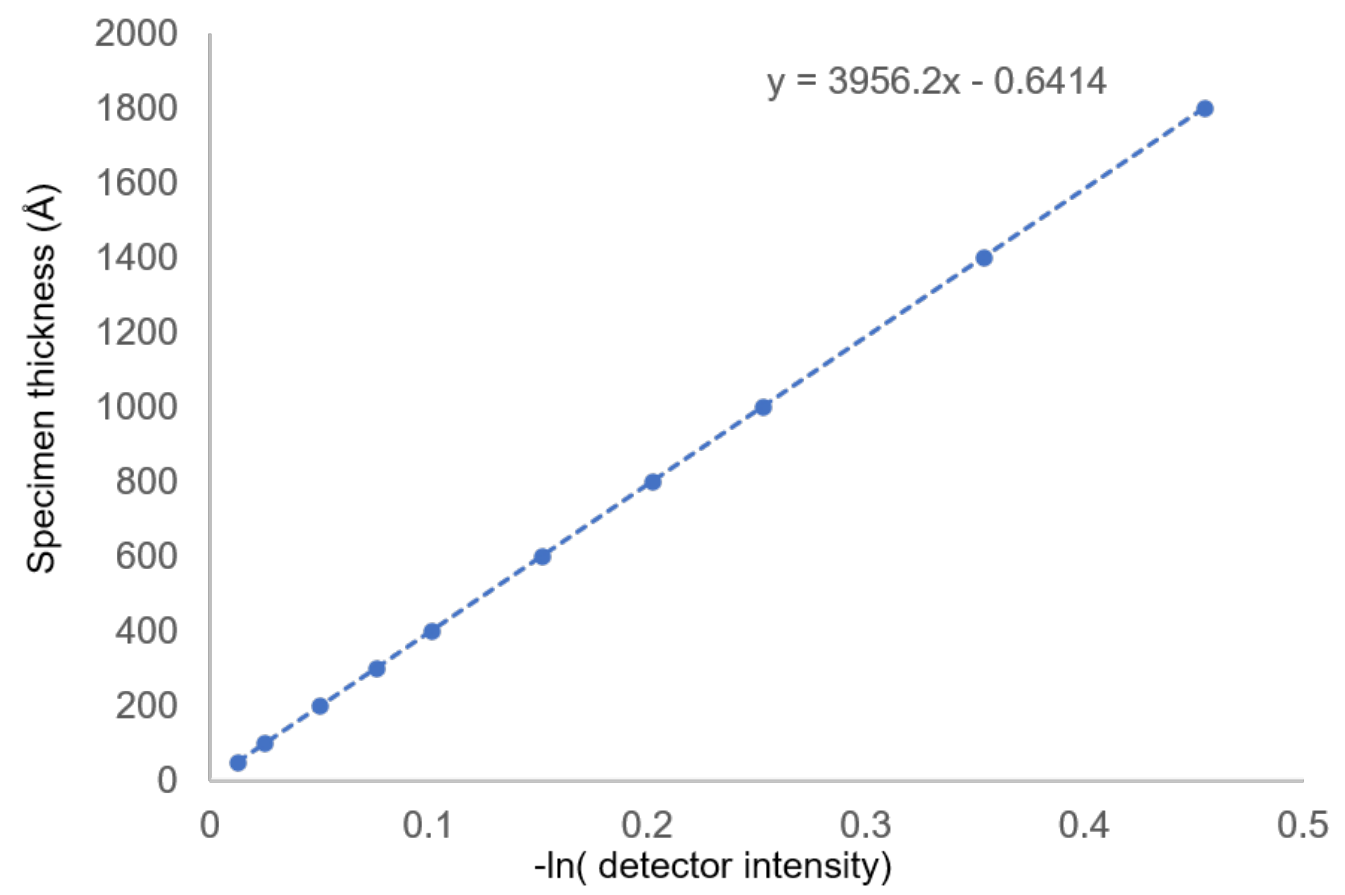

Figure 6 - The coarse-grained all-atom solvent model in combination with the inelastic scattering factor for plasmons we derived produce amplitude losses via the complex potential that do not need to be scaled post hoc. The slope is a read out for the inelastic (single-scatter) mean free path in our simulated solvent. 


\section{Discussion:}

458

459

460

461

462

463

464

465

466

467

468

469

470

471

472

473

474

475

476

477

478

479

480

481

482

483

484

485

486

487

488

489

490

491

492

493
Our simulator implements the most thorough forward model for calculating the interaction between high-energy electrons and radiation-sensitive biological samples demonstrated to date. The improvements described here result from an approximate description of the changes in the specimen due to deposition of energy via inelastic scattering during imaging. This added accuracy in simulating the molecular density produces more realistic image simulations for algorithmic development, but just as importantly, it provides a means to investigate the behavior of complex biological specimens in atomic detail using matched filtering via 2D template matching.

Since the output of the matched filter is sensitive to the spectral distribution of the signal, we can quantify the accuracy of our image formation/damage model by measuring the change in $S N R_{m f}$. We found that modeling the water envelop, detector MTF, residual motion blur, and PDB model uncertainty resulted in a higher $S N R_{m f}$ than could be obtained by optimizing a single B-factor. This analysis is limited by the fact that we cannot strictly disentangle changes to the signal from different envelops that could be mutually compensatory, though this may not be too severe a problem given the differences in the envelopes shown in Figure 3A. A more careful consideration of the impact of different spatial frequencies on $S N R_{m f}$ may prove useful in addressing this limitation in future work.

Our explicit solvent model, while coarse-grained, allows us to accurately reproduce attenuation due to inelastic losses and amplitude contrast that is spatially variable, and based only on the atomic species and local mass thickness in the simulated specimen. In principle, any configuration of atoms can be simulated by supplying an appropriate PDB file to the simulator. In practice, variable solvent thickness, or other sources of structural noise like regions of hexagonal ice could be included directly into the simulator, however, we leave this for future work. We show a considerable improvement in matching $S N R_{\text {solvent }}$ to experimental data and expect this to improve the ability of models (artificial neural networks especially) trained on simulated data to generalize more readily to experimental data. On visual inspection, the granularity in the solvent appears a bit different than that observed in experiment; we suspect including solvated ions might account for this and plan to include this in future work.

Simulations using an explicit solvent model are computationally very demanding, increasing the number of scattering centers to be considered by a factor of $\sim 200$ when simulating single particle image stacks, and up to a factor of $10^{4}$ when simulating micrographs with well-spaced particles. We have addressed this computational demand via multi-threading in $\mathrm{C}++$. Most of the time taken by the wave-propagation calculation is spent on Fourier transforms. The calculation is currently limited to 4 threads per 
simulation, independent of the number of threads used in calculating the real-space potentials. To simulate tilted samples, which will have a substantially larger number of slices to propagate, the Fourier transform can become a bottleneck, suggesting a GPU implementation may be beneficial for future work.

\section{Conclusion:}

Here we have presented an accurate forward model describing sources of signal attenuation and show how the spectral characteristics of that attenuation improve the output of the matched filter $\left(S N R_{m f}\right)$ as used in template matching for the detection of molecules in cryo-EM images. The $S N R_{m f}$ is in turn directly related to the mass limit for detection; any improvement in our forward model results in being able to detect smaller particles, which will expand the capacity of template matching in visual proteomics. The increased $S N R_{m f}$ due to modeling radiation damage is encouraging but should likely be modeled more accurately for the purpose of template matching. We also suggest that our model for inelastic scattering could be improved by direct comparison to experiment using the matched filter. If properly accounted for, we could in principle use this scattering information in particle detection, rather than discarding it using an energy filter.

\section{References}

[1] W. T. Baxter, R. A. Grassucci, H. Gao, and J. Frank, "Determination of signal-tonoise ratios and spectral SNRs in cryo-EM low-dose imaging of molecules," J. Struct. Biol., vol. 166, no. 2, pp. 126-132, May 2009.

[2] S. H. Scheres, R. Nunez-Ramirez, Y. Gomez-Llorente, C. San Martin, P. P. Eggermont, and J.-M. Carazo, "Modeling Experimental Image Formation for Likelihood-Based Classification of Electron Microscopy Data," Structure, no. October, pp. 1167-1177, 2007.

[3] H. Rullgård, L. G. Öfverstedt, S. Masich, B. Daneholt, and O. Öktem, "Simulation of transmission electron microscope images of biological specimens," J. Microsc., vol. 243, no. 3, pp. 234-256, 2011. 
[4] M. Vulovi?? et al., "Image formation modeling in cryo-electron microscopy," J. Struct. Biol., vol. 183, no. 1, pp. 19-32, 2013.

[5] J. M. Cowley and A. F. Moodie, "The scattering of electrons by atoms and crystals. I. A new theoretical approach," Acta Crystallogr., vol. 10, no. 10, pp. 609-619, Oct. 1957.

[6] K. Yonekura, M. B. Braunfeld, S. Maki-Yonekura, and D. A. Agard, "Electron energy filtering significantly improves amplitude contrast of frozen-hydrated protein at 300 kV," J. Struct. Biol., vol. 156, no. 3, pp. 524-536, 2006.

[7] H. P. Erickson, "Fourier transform of an electron micrograph," Adv. opitcal electron Microsc., pp. 163-199, 1973.

[8] H. P. P. Erickson and A. Klug, "Measurement and Compensation of Defocusing and Aberrations by Fourier Processing of Electron Micrographs Author ( $\mathrm{s}$ ): H . P . Erickson and A. Klug Source : Philosophical Transactions of the Royal Society of London . Series B, Biological Sciences, Vol," Philos. Trans. R. Soc. Lond. B. Biol. Sci., vol. 261, pp. 105-118, 1971.

[9] R. F. Egerton, "Measurement of inelastic/elastic scattering ratio for fast electrons and its use in the study of radiation damage," Phys. Status Solidi, vol. 37, no. 2, pp. 663-668, 1976.

[10] L. Reimer and M. Ross-Messemer, "Contrast in the electron spectroscopic imaging mode of a TEM. I. Influence of zero-loss filtering on scattering contrast," J. Microsc., vol. 155, no. 2, pp. 169-182, 1989.

[11] A. J. Noble et al., "Routine single particle CryoEM sample and grid characterization by tomography," Elife, vol. 7, p. e34257, 2018.

[12] Z. Shang and F. J. Sigworth, "Hydration-layer models for cryo-EM image simulation," J. Struct. Biol., vol. 180, no. 1, pp. 10-16, 2012.

[13] G. McMullan, K. R. Vinothkumar, and R. Henderson, "Thon rings from amorphous ice and implications of beam-induced Brownian motion in single particle electron cryo-microscopy," Ultramicroscopy, vol. 158, no. April, pp. 26-32, Apr. 2015.

[14] J. P. Rickgauer, N. Grigorieff, and W. Denk, "Single-protein detection in crowded molecular environments in cryo-EM images," Elife, vol. 6, pp. 1-22, 2017.

[15] R. N. McDonough, Detection of signals in noise, 2nd ed. Academic Press, Inc, 1995.

[16] T. Grant, A. Rohou, and N. Grigorieff, "cis TEM , user-friendly software for singleparticle image processing," pp. 1-24, 2018.

[17] B. A. Lucas, B. A. Himes, L. Xue, T. Grant, J. Mahamid, and N. Grigorieff, 
"Locating Macromolecular Assemblies in Cells by 2D Template Matching with cisTEM," bioarxiv, 2021.

[18] R. F. Egerton, Electron Energy-Loss Spectroscopy in the Electron Microscope. Boston, MA: Springer US, 2011.

[19] R. S. Ruskin, Z. Yu, and N. Grigorieff, "Quantitative characterization of electron detectors for transmission electron microscopy," J. Struct. Biol., vol. 184, no. 3, pp. 385-393, 2013.

[20] P. Tiemeijer, "Titan condenser manual," pp. 1-33, 2005.

[21] M. Vulović, R. B. G. Ravelli, L. J. Van Vliet, A. J. Koster, O. Oktem, and B. Rieger, "Supplementary material associated with the article 'Image Formation Modeling in Cryo-Electron Microscopy ,"' 2013.

[22] E. J. Kirkland, "Image Simulation in Transmission Electron Microscopy," Simulation, pp. 1-14, 2006.

[23] L. Reimer and H. Kohl, Transmission Electron Microscopy, vol. 50, no. 52. 2003.

[24] P. W. Hawkes and E. Kasper, Principles of Electron Optics: Wave Optics, Second. 2018.

[25] K. Ishizuka and N. Uyeda, "A New Theoretical and Practical Approach to the Multislice Method," Acta Crystallogr., vol. 2, pp. 740-749, 1977.

[26] P. Goodman and a. F. Moodie, "Beam Wave Functions in Electron Scattering By the Multi-Slice Method," Acta Crystallogr. Sect. A, vol. 30, no. 2, pp. 280-290, 1974.

[27] J. C. Slater, "Damped electron waves in crystals," Phys. Rev., vol. 51, no. 10, pp. 840-846, 1937.

[28] P. A. Doyle and P. S. Turner, "Relativistic Hartree-Fock X-ray and electron scattering factors," Acta Crystallogr. Sect. A, vol. 24, no. 3, pp. 390-397, 1968.

[29] L. M. Peng, G. Ren, S. L. Dudarev, and M. J. Whelan, "Robust parameterization of elastic and absorptive electron atomic scattering factors," Acta Crystallogr. Sect. A Found. Crystallogr., vol. 52, no. 2, pp. 257-276, 1996.

[30] L. M. Peng, "Electron atomic scattering factors and scattering potentials of crystals," Micron, vol. 30, no. 6, pp. 625-648, 1999.

[31] L. Plante and F. A. Cucinotta, "Cross sections for the interactions of $1 \mathrm{eV}-100$ $\mathrm{MeV}$ electrons in liquid water and application to Monte-Carlo simulation of HZE radiation tracks," New J. Phys., vol. 11, 2009. 
[32] S. L. Dudarev, L. Peng, and M. J. Whelan, "On the Doyle-Turner representation of the optical potential for RHEED calculations," vol. 330, pp. 86-100, 1995.

[33] E. J. Kirkland, "Computation in electron microscopy," Acta Crystallogr. Sect. A Found. Adv., vol. 72, pp. 1-27, 2016.

[34] M. J. Hÿtch and W. M. Stobbs, "Quantitative comparison of high resolution TEM images with image simulations," Ultramicroscopy, vol. 53, no. 3, pp. 191-203, 1994.

[35] M. J. Peet, R. Henderson, and C. J. Russo, "The energy dependence of contrast and damage in electron cryomicroscopy of biological molecules," Ultramicroscopy, vol. 203, no. February, pp. 125-131, 2019.

[36] D. Van Dyck, "Is the frozen phonon model adequate to describe inelastic phonon scattering?," Ultramicroscopy, vol. 109, no. 6, pp. 677-682, 2009.

[37] D. Van Dyck, I. Lobato, F. R. Chen, and C. Kisielowski, "Do you believe that atoms stay in place when you observe them in HREM?," Micron, vol. 68, no. 2014, pp. 158-163, 2015.

[38] H. Fischer, I. Polikarpov, and A. F. Craievich, "Average protein density is a molecular-weight-dependent function," Protein Sci., vol. 13, no. 10, pp. 28252828, 2009.

[39] R. F. Egerton, "Electron energy-loss spectroscopy in the TEM," Reports Prog. Phys., vol. 72, no. 1, 2009.

[40] M. Du and C. Jacobsen, "Relative merits and limiting factors for x-ray and electron microscopy of thick, hydrated organic materials," Ultramicroscopy, vol. 184, no. October, pp. 293-309, 2018.

[41] L. M. Peng, S. L. Dudarev, and M. J. Whelan, High-Energy Electron Diffraction and Microscopy. Oxford University Press, 2010.

[42] M. Wanner, D. Bach, D. Gerthsen, R. Werner, and B. Tesche, "Electron holography of thin amorphous carbon films: Measurement of the mean inner potential and a thickness-independent phase shift," Ultramicroscopy, vol. 106, no. 4-5, pp. 341-345, 2006.

[43] R. Henderson and G. Mcmullan, "Problems in obtaining perfect images by singleparticle electron cryomicroscopy of biological structures in amorphous ice," vol. 62 , no. 1 , pp. 43-50, 2013.

[44] S. B. Hayward and R. M. Glaeser, "Radiation damage of purple membrane at low temperature," Ultramicroscopy, vol. 4, no. 2, pp. 201-210, 1979.

[45] T. Grant and N. Grigorieff, "Measuring the optimal exposure for single particle 
639

640

641

642

643

644

645

646

cryo-EM using a $2.6 \AA$ reconstruction of rotavirus VP6.," Elife, vol. 4, p. e06980, 2015.

[46] A. Bartesaghi et al., "Atomic Resolution Cryo-EM Structure of Article Atomic Resolution Cryo-EM Structure of b -Galactosidase," Struct. Des., vol. 26, no. 6, pp. 848-856.e3, 2018.

[47] S. H. w Scheres, "Beam-induced motion correction for sub-megadalton cryo-EM particles," Elife, vol. 3, p. e03665, 2014.

[48] A. Bartesaghi, D. Matthies, S. Banerjee, A. Merk, and S. Subramaniam, "obtained by cryo-electron microscopy," vol. 2014, 2014.

[49] W. J. Rice et al., "Routine determination of ice thickness for cryo-EM grids," $J$. Struct. Biol., vol. 204, no. 1, pp. 38-44, 2018. 